\title{
Recurrent YAP1-MAML2 and YAP1-NUTM1 fusions in poroma and porocarcinoma
}

\author{
Shigeki Sekine, ${ }^{1,2}$ Tohru Kiyono, ${ }^{3,4}$ Eijitsu Ryo, ${ }^{2}$ Reiko Ogawa, ${ }^{2}$ Susumu Wakai, ${ }^{1}$ Hitoshi Ichikawa, ${ }^{5}$ Koyu Suzuki, ${ }^{6}$ Satoru Arai, \\ Koji Tsuta, ${ }^{8}$ Mitsuaki Ishida, ${ }^{8}$ Yuko Sasajima, ${ }^{9}$ Naoki Goshima, ${ }^{10}$ Naoya Yamazaki, ${ }^{11}$ and Taisuke Mori ${ }^{1,2}$ \\ Department of Diagnostic Pathology, National Cancer Center Hospital, Tokyo, Japan. ${ }^{2}$ Division of Molecular Pathology, ${ }^{3}$ Division of Carcinogenesis and Cancer Prevention, ${ }^{4}$ Department of Cell Culture Technology, \\ and ${ }^{5}$ Department of Clinical Genomics, National Cancer Center Research Institute, Tokyo, Japan. ${ }^{6}$ Department of Pathology and 'Department of Dermatology, St. Luke's International Hospital, Tokyo, Japan. \\ ${ }^{8}$ Department of Pathology and Laboratory Medicine, Kansai Medical University, Osaka, Japan. ${ }^{9}$ Department of Pathology, Teikyo University School of Medicine, Tokyo, Japan. ${ }^{10}$ Molecular Profiling Research \\ Center for Drug Discovery, National Institute of Advanced Industrial Science and Technology, Tokyo, Japan. "Department of Dermatologic Oncology, National Cancer Center Hospital, Tokyo, Japan.
}

\begin{abstract}
Poroma is a benign skin tumor exhibiting terminal sweat gland duct differentiation. The present study aimed to explore the potential role of gene fusions in the tumorigenesis of poromas. RNA sequencing and reverse transcription PCR identified highly recurrent YAP1-MAML2 and YAP1-NUTM1 fusions in poromas (92/104 lesions, $88.5 \%)$ and their rare malignant counterpart, porocarcinomas (7/11 lesions, 63.6\%). A WWTR1-NUTM1 fusion was identified in a single lesion of poroma. Fluorescence in situ hybridization confirmed genomic rearrangements involving these genetic loci. Immunohistochemical staining could readily identify the YAP1 fusion products as nuclear expression of the N-terminal portion of YAP1 with a lack of the C-terminal portion. YAP1 and WWTR1, also known as YAP and TAZ, respectively, encode paralogous transcriptional activators of TEAD, which are negatively regulated by the Hippo signaling pathway. The YAP1 and WWTR1 fusions strongly transactivated a TEAD reporter and promoted anchorage-independent growth, confirming their tumorigenic roles. Our results demonstrate the frequent presence of transforming YAP1 fusions in poromas and porocarcinomas and suggest YAP1/TEADdependent transcription as a candidate therapeutic target against porocarcinoma.
\end{abstract}

\section{Introduction}

Poroma is a skin adnexal tumor exhibiting features of the terminal sweat gland duct $(1,2)$. It typically presents as a solitary sessile nodule on the soles and palms but may occur in any area where sweat glands are present. Histologically, poroma consists of monomorphic small basaloid cells and contains occasional ductal structures. Most commonly, the tumor is broadly connected to the epidermis and grows down into the dermis, forming broad anastomosing bands. Some lesions exhibit a spectrum of architectural variations that allows them to be classified into histological subtypes (Supplemental Figure 1) (2-6). Hidroacanthoma simplex represents an intraepidermal variant, whereas a dermal duct tumor is primarily confined to the dermis. Poroid hidradenoma forms a discrete nodule centered in the dermis and shows characteristic solid and cystic components. Although poromas are relatively common, their genetic background has only been analyzed in a few studies. A recent targeted next-generation sequencing analysis of 6 poromas identified a number of mutations, including HRAS mutations as the sole recurrently mutated gene (7). However, the reported prevalence of HRAS mutations was limited to $17 \%$, suggesting that poromas are a rather heterogeneous group of tumors in terms of genetic alterations $(7,8)$.

Conflict of interest: The authors have declared that no conflict of interest exists. Copyright: (5) 2019, American Society for Clinical Investigation.

Submitted: November 13, 2018; Accepted: May 28, 2019; Published: July 29, 2019

Reference information: J Clin Invest. 2019;129(9):3827-3832.

https://doi.org/10.1172/JCl126185.
Porocarcinoma represents the rare malignant counterpart of poroma predominantly affecting elderly individuals (9-11). In contrast to poromas, porocarcinomas exhibit invasive growth, are prone to local recurrence, and may develop distant metastasis. Because of their rarity and lack of distinct clinical features, the diagnosis of porocarcinoma is often challenging. A subset of porocarcinomas is associated with benign poromas, a finding implying malignant transformation $(2,9,11)$. Furthermore, porocarcinomas arising from longstanding poromas have been reported $(9,12)$. Apart from these, many porocarcinomas do not have a benign precursor component and thus may develop de novo $(11,13)$. A recent sequencing analysis of 5 porocarcinomas identified recurrent mutations in TP53, RB1, EGFR, and HRAS, but simultaneously revealed the molecular diversity of this rare type of tumor (8).

Given that mutations in known oncogenes and tumor suppressor genes are unlikely to underlie the tumorigenesis of a majority of poromas, we used RNA sequencing to explore the possible roles of gene fusions. Our analysis identified highly recurrent YAP1 fusions in poromas and porocarcinomas and demonstrated their transforming activity.

\section{Results and Discussion}

To probe the possible tumorigenic roles of gene fusions in poroma, we subjected 12 formalin-fixed, paraffin-embedded poroma specimens to RNA sequencing. The analysis identified YAP1-MAML2 and the reciprocal MAML2-YAP1 fusions in 4lesions, YAP1-NUTM1 fusions in 3 lesions, and a WWTR1-NUTM1 fusion in 1 lesion (Supplemental Table 1). To expand on this finding, we examined 
A

\begin{tabular}{|c|c|c|c|}
\hline \multicolumn{2}{|c|}{ YAP1-MAML2 fusions } & $\begin{array}{l}\text { Poroma } \\
(n=104)\end{array}$ & $\begin{array}{l}\text { Porocarcinoma } \\
\quad(n=11)\end{array}$ \\
\hline YAP1 exon 1 & MAML2 exon 2-5 & 16 (15.4\%) & 1 (9.1\%) \\
\hline YAP1 exon $1-5$ & MAML2 exon 2-5 & \multirow{2}{*}{55 (52.9\%) } & \multirow{2}{*}{0} \\
\hline YAP1 exon 1-6 & MAML2 exon 2-5 & & \\
\hline
\end{tabular}

MAML2-YAP1 fusions

\begin{tabular}{|c|c|c|}
\hline MAML2 exon 1 & YAP1 exon 2-9 & $9(8.7 \%)$ \\
\hline MAML2 exon 1 & YAP1 exon 7-9 & $39(37.5 \%)$ \\
\hline
\end{tabular}

YAP1-NUTM1 fusions

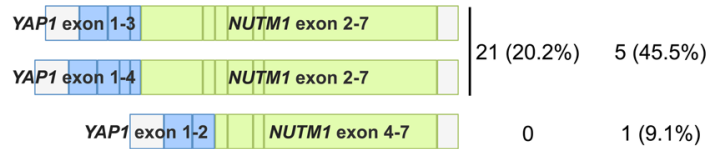

WWTR1-NUTM1 fusion

WWTR1 exon 1-3

NUTM1 exon 2-7

$1(1.0 \%)$

C
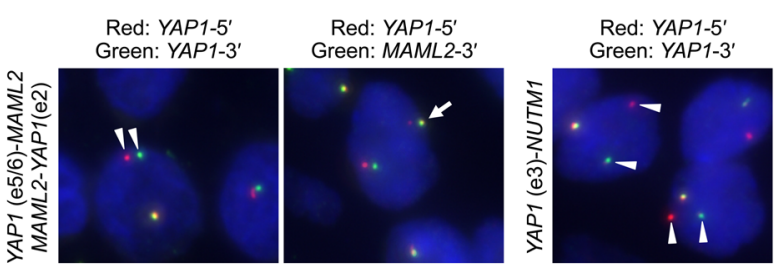

B

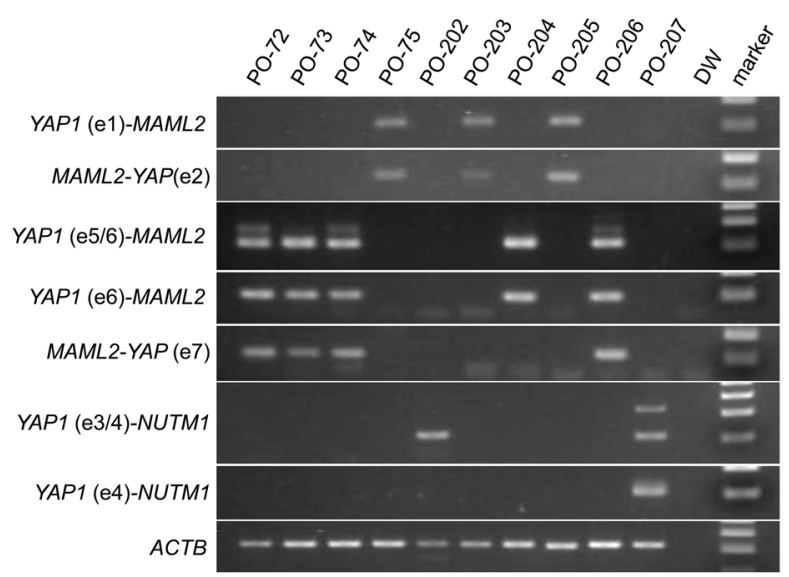

Figure 1. Detection of YAP1 and WWTR1 fusions in poromas and porocarcinomas. (A) Fusion gene transcripts detected in poromas and porocarcinomas. All of the MAML2-YAP1 fusions were associated with the reciprocal YAP1-MAML2 fusions. (B) Representative gel images of RT-PCR products. MAML2-YAP1 fusion transcripts were consistently associated with the reciprocal YAP1-MAML2 fusions. Many of the tumors expressed multiple transcriptional variants. ACTB served as a positive control. (C) Representative fluorescence in situ hybridization images. The detected fusion transcripts are indicated on the left of the respective images. Arrowheads, split signals. Arrows, fused signals.

104 poromas, including those analyzed by RNA sequencing, for the presence of the fusions detected by next-generation sequencing and possible fusion transcripts predicted from the exon structures of YAP1, MAML2, and NUTM1 (Supplemental Table 2). RT-PCR followed by Sanger sequencing detected YAP1-MAML2, MAML2-YAP1, YAP1-NUTM1, and WWTR1-NUTM1 fusions in $71,48,21$, and 1 poromas, respectively (Figure 1, A and B, Supplemental Figure 2). Furthermore, an examination of 11 porocarcinomas identified YAP1-MAML2 and YAP1-NUTM1 fusions in 1 and 6 lesions, respectively. All the lesions with MAML2-YAP1 fusions concurrently expressed the reciprocal YAP1-MAML2 fusions; conversely, 48 of 71 (67.6\%) YAP1-MAML2-positive lesions expressed MAML2-YAP1 fusions. The YAP1-NUTM1 and WWTR1-NUTM1 fusions were mutually exclusive with others. Multiple variant transcripts were detected for YAP1-MAML2, MAML2-YAP1, and YAP1-NUTM1, often in single tumors. All the fusion transcripts were in-frame. Collectively, YAP1 fusions were expressed in 92 poromas $(88.5 \%)$ and 7 porocarcinomas $(63.6 \%)$, and 1 poroma was associated with a WWTR1 fusion (1.0\%). The common presence of YAP1 fusions in poromas and porocarcinomas supports their histogenetic relationship, which is consistent with the idea that a significant proportion of porocarcinomas develop through the malignant transformation of preexisting poromas $(2,9,11)$. To our knowledge, among the identified YAP1 and WWTR1 fusions, only a YAP1-MAML2 fusion was previously reported in a single case of nasopharyngeal carcinoma (14), whereas the other fusion transcripts have not been previously described.
Interestingly, there appear to be associations between specific YAP1 fusion types and clinicopathological features. Hidroacanthoma simplex predominantly expressed the YAP1 exon 1-MAML2 fusion (4/5 lesions). YAP1-NUTM1 fusions were enriched in dermal duct tumors ( $2 / 2$ lesions), poroid hidradenomas ( $4 / 6$ lesions), porocarcinomas (6/11 lesions), poromas in the head and neck region $(15 / 27$ lesions), and poromas with necrotic foci (10/15 lesions). Because the number of each histological variant is rather limited in this study due to their rarity, further investigation based on a larger case series is desirable. However, it is notable that YAP1-NUTM1 fusions are enriched in porocarcinomas and poromas with primarily dermal localization, implying the link between the specific fusions and growth patterns. Examination of other skin tumors, including 24 squamous cell carcinomas, 32 basal cell carcinomas, 5 cutaneous adenocarcinomas, 9 Merkel cell carcinomas, and 27 seborrheic keratoses, did not identify any of the recurrent YAP1 fusions. These observations indicate that YAP1 fusions are highly recurrent and specific to poromas and porocarcinomas among skin neoplasms.

Considering that YAP1 and MAML2 are on the opposite strands of adjacent genetic loci on chromosome 11p, the frequent coexpression of YAP1-MAML2 and MAML2-YAP1 fusion transcripts is thought to be the consequence of intrachromosomal inversions (Supplemental Figure 3). Since NUTM1 and WWTR1 are on chromosome 15q14 and 3q25, respectively, the YAP1-NUTM1 and WWTR1-NUTM1 fusions are expected to result from interchromosomal translocations. Fluorescence in situ hybridization analysis revealed the YAP1 locus rearrange- 


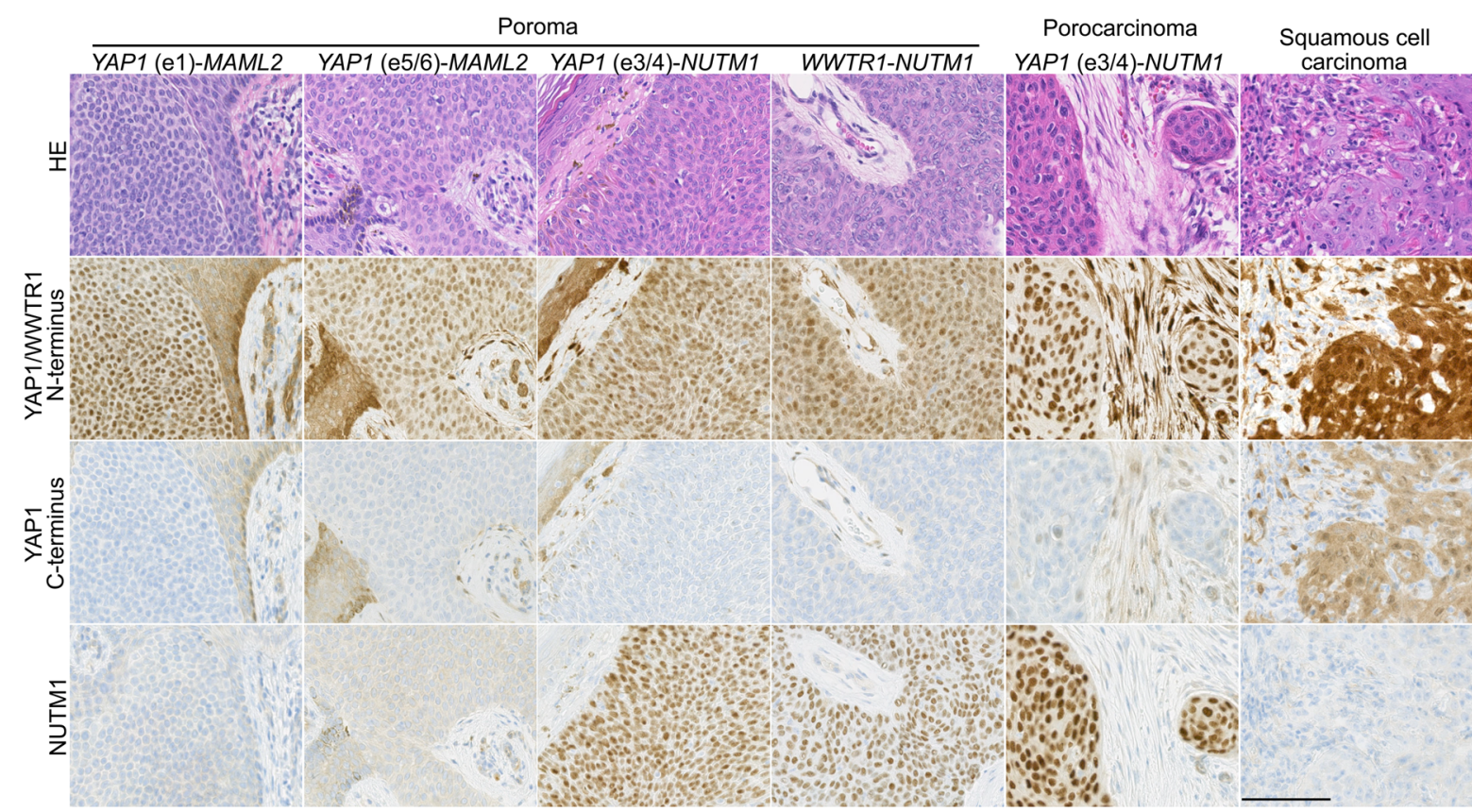

Figure 2. Expression of YAP1 and NUTM1 in poromas, a porocarcinoma, and a squamous cell carcinoma. Tumors with YAP1 fusions showed diffuse nuclear expression of the $\mathrm{N}$-terminal region of YAP1 but lacked the expression of the C-terminal region. Squamous cell carcinoma showed nuclear and cytoplasmic staining with both $\mathrm{N}$ - and C-terminus antibodies. The diffuse nuclear expression of NUTM1 was exclusively observed in tumors with the YAP1-NUTM1 fusions. The anti-YAP1 N-terminus antibody, but not the C-terminus antibody, cross-reacts with the WWTR1. Scale bar: $100 \mu \mathrm{m}$.

ment in 22 of 23 lesions with a YAP1 fusion and the NUTM1 locus rearrangement in 14 of 15 lesions with a NUTM1 fusion (Figure 1C, Supplemental Table 3). Most lesions with reciprocal YAP1MAML2 and MAML2-YAP1 transcripts showed narrow split signals in the assays using YAP1-5' and YAP1-3' probes, consistent with intrachromosomal rearrangement. The YAP1-MAML2 and YAP1-NUTM1 fusion signals were also detected in the tumors with their respective fusion transcripts. Rearrangements of the WWTR1 and NUTM1 loci were confirmed in the lesion with the WWTR1-NUTM1 fusion.

Although there were some differences in the prevalence of respective fusion types, YAP1 fusions were frequently detected in both poromas and porocarcinomas. Therefore, we wanted to find out if any genetic alterations were involved in the malignant transformation of poroid neoplasms. To address this issue, we analyzed 23 poromas and 9 porocarcinomas using the NCC oncopanel test, a next-generation sequencing panel targeting 114 cancer-associated genes (15). The analysis identified a total of 49 protein-altering mutations (Supplemental Table 4 and Supplemental Table 5). Porocarcinomas tended to harbor a larger number of mutations than poromas (average 3.0 vs. 0.96 mutations/ lesion). However, only 3 genes were recurrently mutated in porocarcinomas: KRAS, SETD2, and TP53. Each of these genes was mutated in only 2 of the lesions and SETD2 and TP53 mutations were also detected in poromas. These results indicate that mutations in major cancer-related genes cannot be used to distinguish benign and malignant poroid neoplasms.

To examine the expression of fusion protein products in clinical specimens, we performed immunohistochemical staining using 2 antibodies against YAP1. The analysis revealed the diffuse nuclear expression of the $\mathrm{N}$-terminal portion and the absence of $\mathrm{C}$-terminal portion expression in 100 poromas (96.2\%) and 7 porocarcinomas (63.6\%), including all the lesions with the YAP1 fusions (Figure 2 and Figure 3). The discrepant expression of the N- and C-terminal portions of YAP1 is consistent with the presence of YAP1-MAML2 or YAP1-NUTM1 fusion products. At the same time, it suggests the absence of MAML2-YAP1 fusion products. Seven poromas showed the discordant expression of $\mathrm{N}$ - and C-terminal regions of YAP1 in the absence of YAP1 fusion transcripts. We expect that these lesions might also have different types of YAP1 fusions, but the limitation in sample quality due to the use of formalin-fixed paraffin-embedded specimens precluded further investigation of other fusions in these lesions. The lesion with the WWTR1-NUTM1 fusion was also diffusely positive for the YAP1 N-terminus antibody, likely reflecting the known cross-reactivity of the antibody with the N-terminal portion of WWTR1 (16). Expression of NUTM1, also known as NUT, was observed in 25 poromas (24.0\%) and 6 porocarcinomas $(54.5 \%)$, including all lesions with the YAP1-NUTM1 fusions.

YAP1 immunohistochemistry on other skin tumors, including 23 squamous cell carcinomas, 24 basal cell carcinomas, 5 cutaneous adenocarcinomas, 9 Merkel cell carcinomas, and 26 seborrheic keratoses, showed common expression of YAP1, in agreement with previous reports $(17,18)$. However, in contrast to poromas and porocarcinomas, the expression patterns of the $\mathrm{N}$ - and $\mathrm{C}$-terminal regions of YAP1 were consistently identical in the respective tumors, consistent with the WT protein expression. Also, they exhibited cytoplasmic in addition to nuclear expression of YAP1, unlike poromas and porocarcinomas. Thus, our immunohistochemical analysis could readily distinguish YAP1-MAML2 and YAP1-NUTM1 fusion products from the WT YAP1 based on the discordant expression of the $\mathrm{N}$ - and C-terminal regions of YAP1. Additionally, NUTM1 expression was never observed in skin tumors other than poromas and porocarcino- 


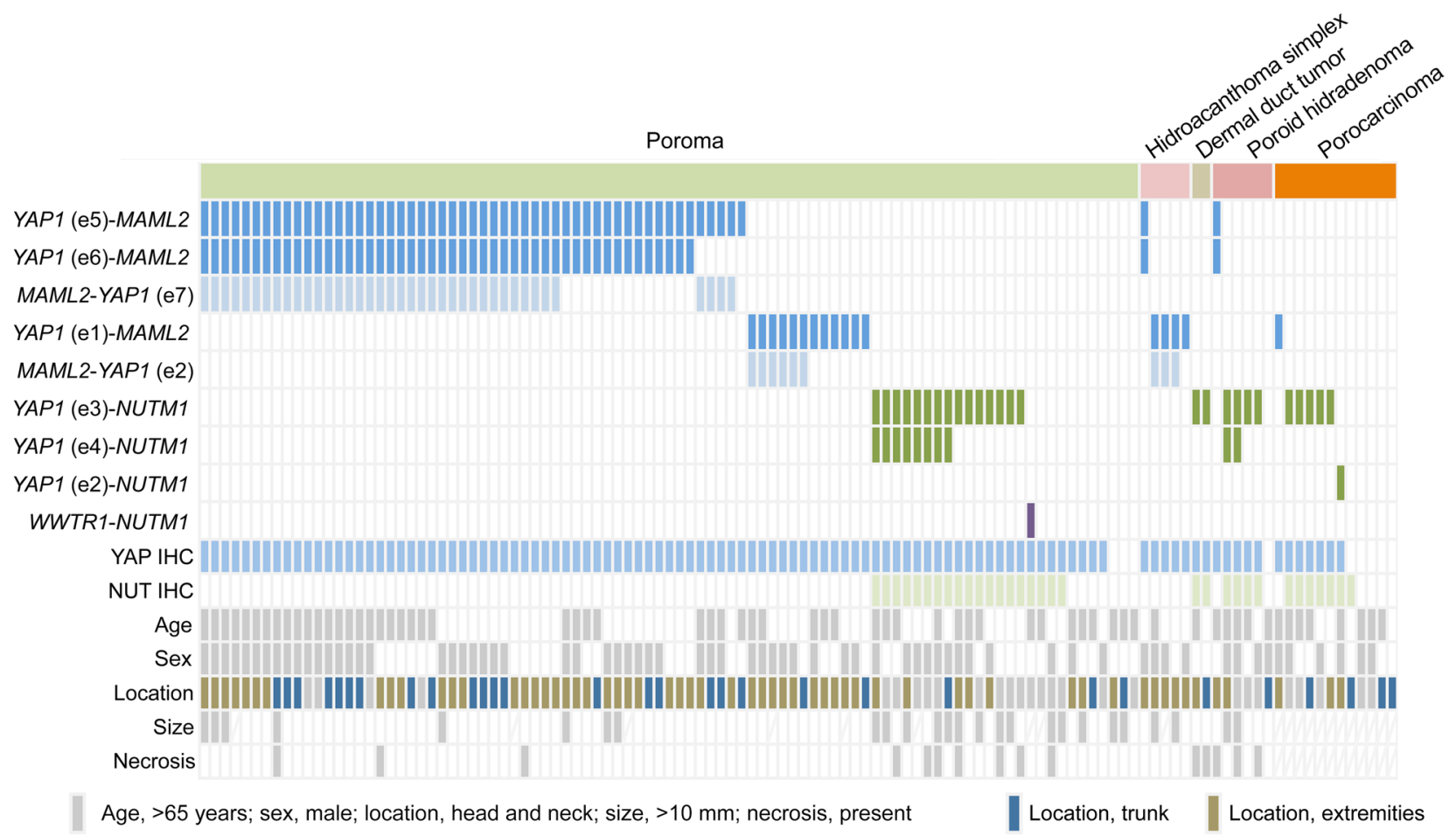

Figure 3. Summary of fusion transcript expression, immunohistochemical results, and clinicopathological features of poroma and porocarcinoma. Poroma $(n=104)$; porocarcinoma $(n=11)$. YAP1 immunohistochemistry (IHC) indicates cases with discordant expression of the N-and C-terminal regions.

mas, indicating its specificity for NUTM1 fusion-positive poromas and porocarcinomas among skin tumors. Taken together, YAP1 and WWTR1 fusions were exclusive to poromas and porocarcinomas among skin tumors, and thus the detection of these fusion transcripts or protein products would have diagnostic applications.

The identification of a WWTR1 fusion in a single lesion of poroma, in addition to frequent YAP1 fusions, is remarkable because both YAP1 and WWTR1, also known as YAP and TAZ, respectively, encode paralogous transcriptional regulators of $\operatorname{TEAD}(19,20)$. Activation of YAP1/WWTR1 is widely observed in various tumorigenic processes and plays roles in stemness, proliferation, invasion, and chemoresistance $(19,21)$. Physiologically, the activity of YAP1/WWTR1 is negatively regulated by the Hippo signaling pathway. Inactivating mutations of Hippo pathway components, leading to aberrant activation of YAP1/WWTR1, have been reported in various cancers, supporting the tumor suppressive function of this pathway $(19,20)$. Recurrent genetic alterations of YAP1 and WWTR1 themselves have only been reported in epithelioid hemangioendothelioma (22-24). More than 90\% of epithelioid hemangioendotheliomas have WWTR1-CAMTA1 fusions and most of the remaining cases express a YAP1-TFE3 fusion. The WWTR1-CAMTA1 fusion protein shows constitutive nuclear localization, dysregulated TEAD activation, and transforming activity (25). Notably, similar to these previously reported WWTR1 and YAP1 fusions, the putative YAP1-MAML2, YAP1-NUTM1, and WWTR1-NUTM1 fusion products retain TEAD-binding domains derived from YAP1 or WWTR1 (Figure 4A).

MAML2 constitutes the 3 ' part of CRTC1-MAML2 fusions, which is common in mucoepidermoid carcinoma of the salivary gland, and the interaction between p300 and the MAML2-derived transactivation domain is critical for their transforming activity $(26,27)$. The
NUTM1-derived portion of YAP1-NUTM1 fusions also constitutes other tumor-related fusion proteins, including BRD4-NUTM1 in NUT midline carcinoma and CIC-NUTM1 in central nervous system Ewing sarcoma family tumor (28-30), and the recruitment of CBP and p300 is required for the oncogenic property of the BRD4-NUTM1 fusion (29). Thus, all the YAP1 and WWTR1 fusions detected in poromas and porocarcinomas harbor the N-terminal TEAD-binding domain of YAP1 or WWTR1 (31), and the MAML2- or NUTM1-derived regions that interact with transcriptional coactivators, $\operatorname{CBP}$ and $\mathrm{p} 300(27,29)$. Based on the common presence of domains to interact with transcription factors and transcriptional cofactors across these fusions, we postulated that these fusions function as a potent transcriptional activator of TEAD. In contrast, MAML2-YAP1 fusions lack these domains.

To test the functional significance of the YAP1 fusions, we first examined the intracellular localization of the YAP1 fusion products in HEK293T cells. Normally, active Hippo pathway signaling leads to the cytoplasmic retention and degradation of YAP1, inhibiting the formation of a complex with TEAD transcription factors $(19,20)$. WT YAP1 and a constitutively active mutant, $\mathrm{YAP}^{\mathrm{S127A}}$, were distributed in both the nucleus and cytoplasm, with a predominant localization in the cytoplasm (Figure 4B). In contrast, YAP1-MAML2 and YAP1NUTM1 fusions exhibited exclusively nuclear localization with speckled and homogeneous staining patterns, respectively, suggesting that the 3 '-fusion partners contribute to the nuclear localization of these fusions through distinct mechanisms. Next, we performed a reporter assay to test the role of the fusions in TEAD-dependent transcription. As expected, YAP1-MAML2, YAP1-NUTM1, and WWTR1-NUTM1 fusions strongly upregulated the expression of a TEAD reporter, and the reporter expression levels were even higher than those with 2 YAP1 mutants that are resistant to negative regu- 
A

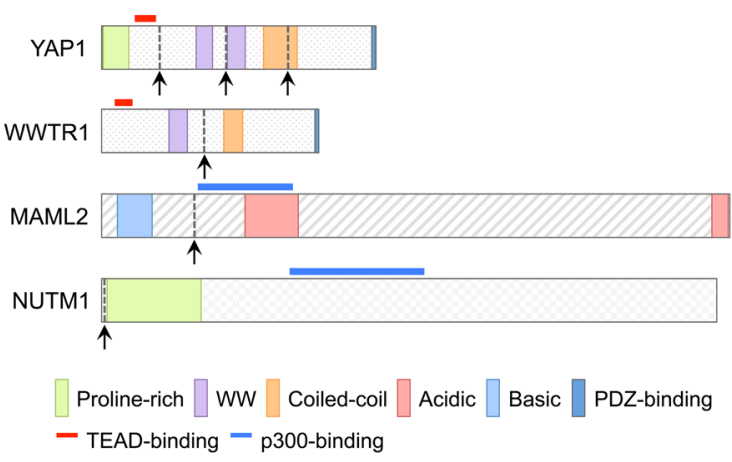

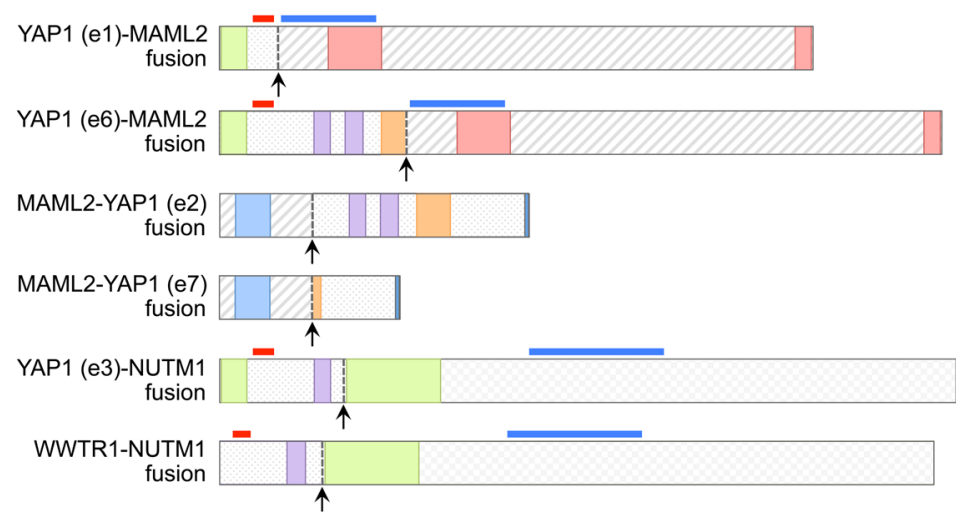

B
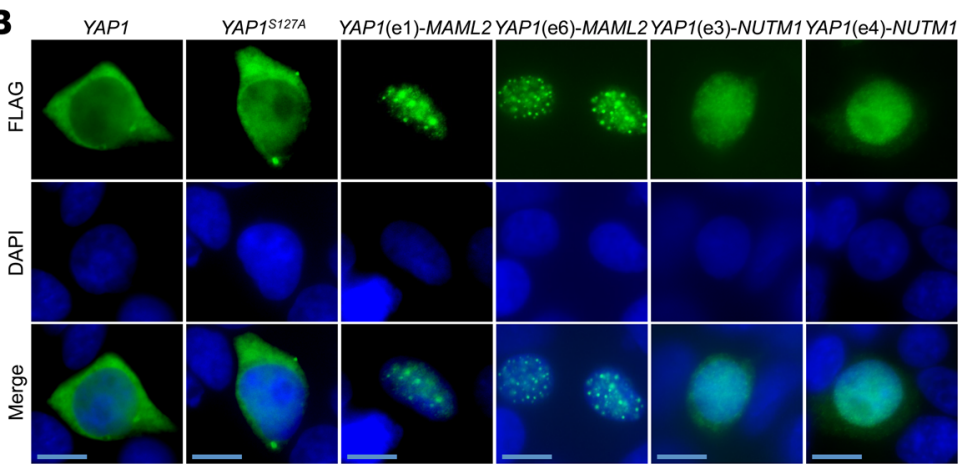

C

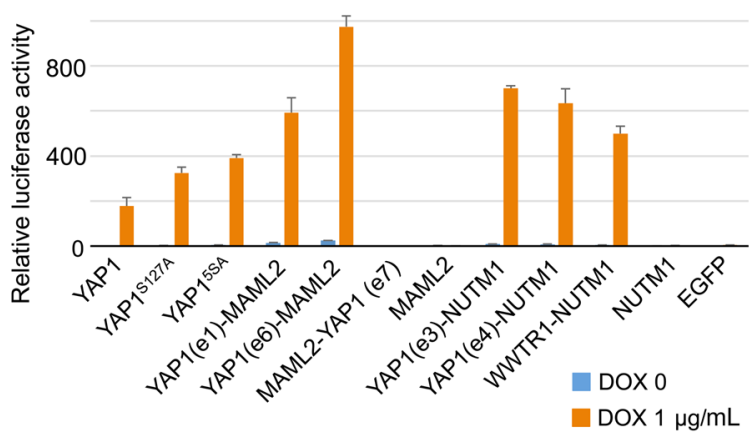

D
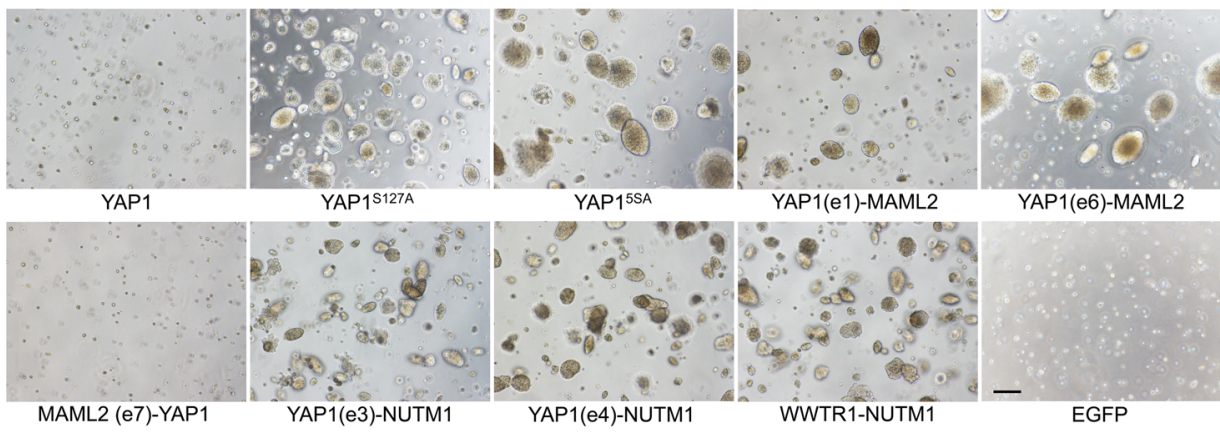

Figure 4. Functional significance of YAP1 and WWTR1 fusions. (A) Structures of the putative fusion gene products expressed in poromas and porocarcinomas. Arrows indicate fusion breakpoints. Notice that YAP1-MAML2, YAP1-NUTM1, and WWTR1-NUTM1 fusion gene products harbor a TEAD-binding domain derived from YAP1 or WWTR1 and a p300-binding domain derived from MAML2 or NUTM1. (B)Intracellular localization of YAP1 fusion gene products. The FLAG-tagged proteins were detected by immunofluorescence staining with nuclear staining using DAPI. Scale bars: $10 \mu \mathrm{m}$. (C) TEAD luciferase reporter assays on HEK293T cells transfected with doxycycline-inducible vectors. YAP1 ${ }^{\text {S127A }}$ and YAP1 ${ }^{\text {SSA }}$ represent constitutively active mutants. The luciferase activity of EGFP-transfected cells in the absence of doxycycline was set at 1 to indicate relative luciferase activities. Data represent mean of triplicate measurements \pm SD. (D) Soft agar colony formation assay of NIH3T3 cells expressing the fusion transgenes. NIH3T3 cells were transduced with doxycycline-inducible retrovirus vectors to express the respective transgenes and subjected to a soft agar colony formation assay in the presence of doxycycline. None of the clones formed colonies in the absence of doxycycline. Scale bar: $200 \mu \mathrm{m}$.

lation by the Hippo pathway. In contrast, the MAML2-YAP1 fusion, MAML2, and NUTM1 did not significantly induce the TEAD reporter expression (Figure 4C).

We then established a series of doxycycline-dependent inducible NIH3T3 cell lines. Despite repeated attempts, we could not obtain sublines with high expression levels of YAP1-MAML2 fusions (Supplemental Figure 4). However, in a soft agar colony formation assay, the induction of YAP1-MAML2, YAP1-NUTM1, and WWTR1NUTM1 expression resulted in anchorage-independent growth, similar to that seen in constitutively active YAP1 mutants (Figure 4D) (32). In contrast, the wild-type YAP1 and the MAML2-YAP1 fusion did not promote anchorage-independent growth. These observations indicate the role of YAP1 and WWTR1 fusions in the activa- tion of TEAD transcription factors and their transforming activity. Although two-thirds of YAP1-MAML2 fusions were associated with the reciprocal MAML2-YAP1 fusions, functional assays showed the lack of their transforming activity, and immunohistochemical study failed to detect their protein products. Thus, MAML2-YAP1 fusions are likely nonfunctional by-products of YAP1-MAML2 fusions, resulting from intrachromosomal inversion.

Next, we tested if YAP1 and WWTR1 fusions also show transforming activity in epithelial cells by using an immortalized human dermal keratinocyte (HDK) cell line (33). A reporter assay was performed by using doxycycline-dependent inducible cell lines stably transduced with a TEAD reporter (Supplemental Figure 5A), because of the low transfection efficiency of the HDK cells. As 
observed with NIH3T3 cells, expression of YAP1-MAML2, YAP1NUTM1, and WWTR1-NUTM1 fusions strongly upregulated the expression of the TEAD reporter (Supplemental Figure 5B). A soft agar colony formation assay showed enhanced anchorage-independent growth upon the induction of YAP1-MAML2, YAP1-NUTM1, and WWTR1-NUTM1 (Supplemental Figure 5C). However, these colonies were smaller than those formed by cells expressing constitutively active YAP1 and WWTR1 mutants. In addition, cells overexpressing WT YAP1 and WWTR1 also formed small colonies. These findings indicate that YAP1 and WWTR1 fusions activate TEAD transcription factors and also promote anchorage-independent growth in epithelial cells. However, they may exhibit somewhat different biological effects in different cell types.

The present study identified highly recurrent YAP1 and WWTR1 fusions in poromas and porocarcinomas. The YAP1 and WWTR1 fusions are potent transcriptional activators of TEAD and have transforming activity. The respective fusion types are likely associated with several clinicopathological features, including the enrichment of YAP1-NUTM1 fusions in porocarcinomas. Since YAP1 fusions are specific to poromas and porocarcinomas among skin tumors, the detection of their protein products would have immediate diagnostic applications. Furthermore, the identification of recurrent YAP1 fusions in porocarcinomas suggests YAP1/ TEAD-dependent transcription as a potential therapeutic target against this rare malignancy.

\section{Methods}

Additional information is provided in Supplemental Methods.

Study approval. The study was approved by the Ethical Committee of National Cancer Center, Tokyo, Japan (approval 2017-078).

\section{Author contributions}

SS, TK, and TM contributed equally to this work. SS, TK, and TM designed the study. SS, ER, RO, HI, TM performed next-generation and Sanger sequencing. SS, SW, and TM performed histological, immunohistochemical, and FISH analyses. TK performed functional assays. KS, SA, KT, MI, YS, and NY provided tissue samples and clinical information. NG provided cDNA clones. SS wrote the manuscript with support from TK and TM.

\section{Acknowledgments}

TK is supported by Grants-in-Aid for the National Cancer Center Research and Development Fund (29-E-5) and the Japan Society for the Promotion of Science KAKENHI (16H04701). The authors thank T. Ishiyama, K. Tanaka, C. Kohno, S. Miura, T. Sakaguchi, and C. Kina for expert technical assistance.

Address correspondence to: Shigeki Sekine, Tohru Kiyono, or to Taisuke Mori, National Cancer Center Hospital, 5-1-1, Tsukiji, Chuo-ku, Tokyo 104-0045, Japan. Phone: 81.3.3542.2511; Email: ssekine@ ncc.go.jp (SS); tkiyono@ncc.go.jp (TK); tamori@ncc.go.jp (TM).
1. Goldman P, Pinkus H, Rogin JR. Eccrine poroma; tumors exhibiting features of the epidermal sweat duct unit. AMA Arch Derm. 1956;74(5):511-521.

2. Abenoza P, Ackerman AB. Poroma. Neoplasms with eccrine differentiation. Philadelphia: Lea and Febiger; 1990:113-185.

3. Hu CH, Marques AS, Winkelmann RK. Dermal duct tumor: a histochemical and electron microscopic study. Arch Dermatol. 1978;114(11):1659-1664.

4. Rahbari H. Hidroacanthoma simplex--a review of 15 cases. Br J Dermatol. 1983;109(2):219-225.

5. Liu HN, Chang YT, Chen CC, Huang CH. Histopathological and immunohistochemical studies of poroid hidradenoma. Arch Dermatol Res. 2006;297(7):319-323.

6. Battistella M, Langbein L, Peltre B, Cribier B. From hidroacanthoma simplex to poroid hidradenoma: clinicopathologic and immunohistochemic study of poroid neoplasms and reappraisal of their histogenesis. Am J Dermatopathol. 2010;32(5):459-468.

7. Bosic M, et al. Targeted molecular profiling reveals genetic heterogeneity of poromas and porocarcinomas. Pathology. 2018;50(3):327-332.

8. Harms PW, et al. Porocarcinomas harbor recurrent HRAS-activating mutations and tumor suppressor inactivating mutations. Hum Pathol. 2016;51:25-31.

9. Shaw M, McKee PH, Lowe D, Black MM. Malignant eccrine poroma: a study of twenty-seven cases. Br J Dermatol. 1982;107(6):675-680.

10. Mehregan AH, Hashimoto K, Rahbari H. Eccrine adenocarcinoma. A clinicopathologic study of 35 cases. Arch Dermatol. 1983;119(2):104-114.

11. Robson A, et al. Eccrine porocarcinoma (malignant eccrine poroma): a clinicopathologic study of 69 cases. Am J Surg Pathol. 2001;25(6):710-720.

12. Puttick L, Ince P, Comaish JS. Three cases of eccrine porocarcinoma. Br J Dermatol. 1986;115(1):111-116.
13. Lozano Orella JA, Valcayo Peñalba A, San Juan CC, Vives Nadal R, Castro Morrondo J, Tuñon Alvarez T. Eccrine porocarcinoma. Report of nine cases. Dermatol Surg. 1997;23(10):925-928.

14. Valouev A, et al. Discovery of recurrent structural variants in nasopharyngeal carcinoma. Genome Res. 2014;24(2):300-309.

15. Sunami K, et al. Feasibility and utility of a panel testing for 114 cancer-associated genes in a clinical setting: A hospital-based study. Cancer Sci. 2019;110(4):1480-1490.

16. Furukawa KT, Yamashita K, Sakurai N, Ohno S. The Epithelial Circumferential Actin Belt Regulates YAP/TAZ through Nucleocytoplasmic Shuttling of Merlin. Cell Rep. 2017;20(6):1435-1447.

17. Debaugnies M, et al. YAP and TAZ are essential for basal and squamous cell carcinoma initiation. EMBO Rep. 2018;19(7):e45809.

18. Maglic D, et al. YAP-TEAD signaling promotes basal cell carcinoma development via a c-JUN/ AP1 axis. EMBO J. 2018;37(17):e98642.

19. Harvey KF, Zhang X, Thomas DM. The Hippo pathway and human cancer. Nat Rev Cancer. 2013;13(4):246-257.

20. Varelas X. The Hippo pathway effectors TAZ and YAP in development, homeostasis and disease. Development. 2014;141(8):1614-1626.

21. Zanconato F, Cordenonsi M, Piccolo S. YAP/ TAZ at the Roots of Cancer. Cancer Cell. 2016;29(6):783-803.

22. Errani C, et al. A novel WWTR1-CAMTA1 gene fusion is a consistent abnormality in epithelioid hemangioendothelioma of different anatomic sites. Genes Chromosomes Cancer. 2011;50(8):644-653.

23. Tanas MR, et al. Identification of a disease-defining gene fusion in epithelioid hemangioendothelioma. Sci Transl Med. 2011;3(98):98ra82.
24. Antonescu CR, et al. Novel YAP1-TFE3 fusion defines a distinct subset of epithelioid hemangioendothelioma. Genes Chromosomes Cancer. 2013;52(8):775-784.

25. Tanas MR, et al. Mechanism of action of a WWTR1(TAZ)-CAMTA1 fusion oncoprotein. Oncogene. 2016;35(7):929-938.

26. Tonon G, et al. t(11;19) (q21;p13) translocation in mucoepidermoid carcinoma creates a nove fusion product that disrupts a Notch signaling pathway. Nat Genet. 2003;33(2):208-213.

27. Wu L, et al. Transforming activity of MECT1MAML2 fusion oncoprotein is mediated by constitutive CREB activation. EMBO J. 2005;24(13):2391-2402.

28. French CA, Miyoshi I, Kubonishi I, Grier HE, Perez-Atayde AR, Fletcher JA. BRD4-NUT fusion oncogene: a novel mechanism in aggressive carcinoma. Cancer Res. 2003;63(2):304-307.

29. Reynoird N, et al. Oncogenesis by sequestration of $\mathrm{CBP} / \mathrm{p} 300$ in transcriptionally inactive hyperacetylated chromatin domains. EMBOJ. 2010;29(17):2943-2952.

30. Sturm D, et al. New brain tumor entities emerge from molecular classification of CNS-PNETs. Cell. 2016;164(5):1060-1072.

31. Hau JC, et al. The TEAD4-YAP/TAZ protein-protein interaction: expected similarities and unexpected differences. Chembiochem. 2013;14(10):1218-1225

32. Zhao B, et al. Inactivation of YAP oncoprotein by the Hippo pathway is involved in cell contact inhibition and tissue growth control. Genes Dev. 2007;21(21):2747-2761.

33. Egawa N, et al. The E1 protein of human papillomavirus type 16 is dispensable for maintenance replication of the viral genome. J Virol. 2012;86(6):3276-3283. 